\title{
CircRNAs: role in human diseases and potential use as biomarkers
}

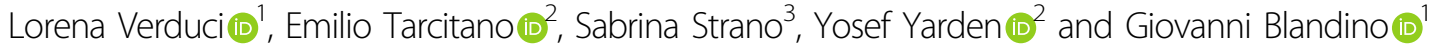

\begin{abstract}
Circular RNAs (circRNAs) are a class of endogenous RNAs characterized by a covalent loop structure. In comparison to other types of RNAs, the abundance of circRNAs is relatively low but due to the circular configuration, their stability is very high. In addition, circRNAs display high degree of tissue specificity. The sponging activity of circRNAs toward microRNAs is the best-described mode of action of circRNAs. However, the ability of circRNAs to bind with specific proteins, as well as to encode short proteins, propose alternative functions. This review introduces the biogenesis of circRNAs and summarizes the roles played by circRNAs in human diseases. These include examples of their functional roles in several organ-specific cancers, such as head and neck and breast and lung cancers. In addition, we review potential functions of circRNAs in diabetes, cardiovascular, and neurodegenerative diseases. Recently, a growing number of studies have demonstrated involvement of circRNAs in a wide spectrum of signaling molecular pathways, but at the same time many different and controversial views on circRNAs role and function are emerging. We conclude by offering cellular homeostasis generated by networks comprising circular RNAs, other non-coding RNAs and RNA-binding proteins. Accordingly, it is predictable that circRNAs, due to their highly stable nature and remarkable tissue specificity, will emerge as reliable biomarkers of disease course and treatment efficacy.
\end{abstract}

\section{Facts}

- circRNAs are single-stranded circles of RNA, which form highly stable closed loops.

- circRNAs can have different functions. Among these, the miRNA sponging is the best-characterized role.

- circRNAs are widely expressed in human tissues and their expression is highly tissue-specific.

- circRNAs are involved in many human diseases, including cancer and neurodegenerative disorders.

- The biochemical characteristics of circRNAs,

Correspondence: Yosef Yarden (yosef.yarden@weizmann.ac.il) or

Giovanni Blandino (giovanni.blandino@ifo.gov.it)

${ }^{1}$ Unit of Oncogenomic and Epigenetic, Department of Research, Advanced Diagnostic, and Technological Innovation, IRCCS, Regina Elena National Cancer Institute, Rome, Italy

${ }^{2}$ Department of Biological Regulation, Weizmann Institute of Science, Rehovot, Israel

Full list of author information is available at the end of the article

Edited by G. Melino especially stability and tissue specificity, make them ideal biomarkers for clinical use.

\section{Open questions}

- Are there yet unknown features controlling circRNA biogenesis?

- Are there still undiscovered functional aspects and mechanisms of circRNAs?

- How do circRNAs function in already wellcharacterized molecular pathways?

- To what extent would deeper understanding and utilization of circRNAs help improve human health?

\section{Introduction}

Circular RNAs (circRNAs) are covalently closed circular RNA molecules recently reconsidered for their important roles in cancer and in other human diseases ${ }^{1-3}$. Since 2013, when Memczak et al. ${ }^{4}$ reported that circRNAs act as post-transcriptional regulators, additional circRNAs have 
been identified, implying an important regulatory potential for this class of molecules. It is currently broadly recognized that circRNAs have significant roles to play in cell proliferation ${ }^{5-7}$, motility and metastasi ${ }^{5-9}$, as well as in cell cycle progression ${ }^{10}$, angiogenesis ${ }^{11,12}$, and apoptosis ${ }^{13}$.

To exemplify the biomedical potential of circRNAs, we briefly review the biogenesis of circRNAs and then describe the role of some circRNAs in head and neck squamous cell carcinoma, breast and lung cancer. Among neurological disorders, we focus herein on Alzheimer disease. In addition, we report examples of circRNAs playing functional roles in cardiovascular diseases and in diabetes. Although the sponging mechanism of circRNAs toward microRNAs has emerged as the most common mechanism of action, additional modes of action have been proposed. CircRNAs can interact with proteins and some are translated into novel polypeptides or act as transcriptional regulators ${ }^{2,14-21}$. Presumably, as many circRNAs are being characterized, additional modes of action will be uncovered soon. CircRNAs are involved in many signaling pathways and some of these molecular pathways have been already characterized for their important roles in human diseases and they are subjects of clinical trials ${ }^{22,23}$. These characteristics, together with their presence in accessible body fluids, such as saliva, blood, and urine, make the circRNAs promising therapeutic targets and potential biomarkers for human diseases $^{24-26}$.

\section{Biogenesis and function of circRNAs}

CircRNAs can derive from exons, introns, antisense, $5^{\prime}$ or $3^{\prime}$ untranslated and intergenic genomic regions ${ }^{27}$. Exonic circRNAs (ecircRNAs) represent the most abundant species and they are produced by a "back-splicing" mechanism. During the biogenesis process, a downstream $5^{\prime}$ splice site of an exon is joined to an upstream $3^{\prime}$ splice site of the same or another exon, involving single or multiple exons ${ }^{1,28-32}$. The molecules derived from this mechanism form a closed circular transcript and an alternatively spliced linear RNA with skipped exons ${ }^{31}$. Thus, the mechanism that generates circRNAs uses the canonical spliceosomal machinery ${ }^{31}$. As a consequence, transcription of circRNAs competes with canonical premRNA splicing and affects the rate of canonical gene expression $^{28}$.

One of the best-described mechanisms explaining the biological function of circRNAs is the ability to effectively sponge microRNAs ${ }^{33}$ (Fig. 1). The circ-SRY ${ }^{33}$, CDR1as $^{4,33,34}$, circ-ITCH ${ }^{35}$, circHIPK3 (ref. ${ }^{36}$ ), circ_000984 (ref. ${ }^{37}$ ), circ-TTBK2 (ref. ${ }^{38}$ ), and circPVT1 (ref. ${ }^{39}$ ) are examples of circRNAs that act as miRNA sponges (also called competing endogenous RNA; ceRNA). CircRNAs function as ceRNA via microRNA

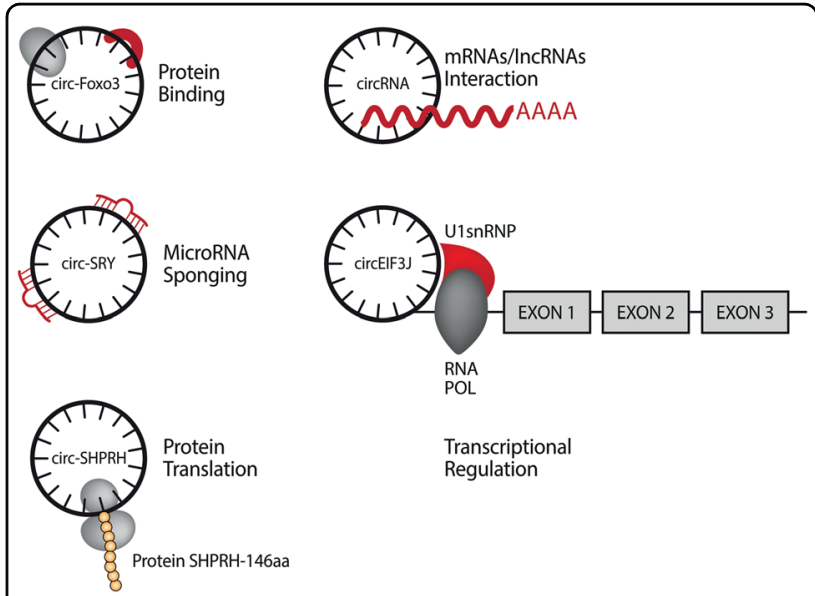

Fig. 1 circRNA functions. CircRNAs can interact with proteins and other RNAs, acting as microRNA sponge, regulate the transcription and in some cases they can also be translated in proteins.

(miRNA) sequestration, by binding to miRNA response elements (MREs) ${ }^{4}$. Each circRNA can have many MREs on the miRNA target and the number of MREs is related to the length of the circRNAs themselves ${ }^{4}$.

CircRNAs can also interact with proteins (Fig. 1). Examples of circRNAs interacting with proteins include circ-PABPN ${ }^{14}$, circ-Foxo3 (ref. ${ }^{10}$ ), and circ-Amotl1 $\left(\right.$ ref. $\left.{ }^{15}\right)$. RNA-binding proteins (RBPs) specifically interact with RNA molecules to form ribonucleoprotein complexes ${ }^{14}$. The RBP HuR can bind circ-PABPN1 in human cervical carcinoma HeLa cells and it is responsible for the translation rate of the PABPN1 gene ${ }^{14}$ (Fig. 1). The RNA-binding protein quaking-5 (QKI-5) promotes circRNA biogenesis during epithelial-to-mesenchymal transition (EMT) through interaction with introns flanking the circRNA-forming exons ${ }^{16}$. Another protein that regulates circRNA biogenesis is the splicing factor called muscleblind (MBL). MBL promotes the formation of circMBL through interaction with introns flanking the circRNA itself ${ }^{28}$.

There are several examples of circRNA-protein interactions in the cancer context (Fig. 1). The tumor suppressor circ-Foxo3 interacts with CDK2 and p21 to form a ternary complex and inhibit cell cycle progression in cancer ${ }^{10}$. The oncogenic circRNA circ-Amotll promotes cell growth through an interaction with the protooncogene $c-M Y C$. Circ-Amotl1 is able to increase the retention of nuclear c-MYC, promote c-myc stability, and up-regulating c-myc targets ${ }^{15}$.

There is also evidence showing that circRNAs can be translated into functional proteins (Fig. 1). Circ-ZNF609 is one of the first examples described of a circRNA that can be translated into a protein. Circ-ZNF609 is involved in the regulation of myoblast proliferation ${ }^{17}$. 
The circular form of the SNF2 histone linker PHD RING helicase (SHPRH) gene, which encodes the protein SHPRH-146aa represents an additional example ${ }^{18}$. Both circ-SHPRH and SHPRH-146aa are highly expressed in normal human brains and their expression was found to be down-regulated in glioblastoma, suggesting a tumor suppressor role ${ }^{18}$. In a similar way, Zheng et al. ${ }^{19}$ identified circPPP1R12A, which is up-regulated in colon cancer (CC) and can be translated into a protein contributing to the rapid proliferation of CC cells via the Hippo-YAP pathway.

Finally, it is well accepted that intronic circRNAs (ciRNAs) and exon-intron circRNAs (ElciRNAs) can act as transcriptional regulators (Fig. 1) ${ }^{20,21}$. The intronic circRNA ci-ankrd52 is able to regulate its parental gene expression by modulating RNA polymerase II's elongation activity $^{21}$. Similarly, two ElciRNAs, circEIF3J and circPAIP2, are able to regulate the expression of their parental genes through a specific RNA-RNA interaction between U1 snRNA and the circRNA ${ }^{20}$. More recently Stoll et al. $^{40}$ showed that the intronic circle ci-Ins2, located mainly in the nucleus of pancreatic $\beta$ cells, is able to regulate insulin secretion through interaction with the TAR DNA-binding protein $43 \mathrm{kDa}$ (TDP-43).

\section{CircRNAs in head and neck squamous cell carcinoma}

Head and neck cancers represent the sixth most common cancer worldwide ${ }^{41,42}$. This cancer usually initiates in the squamous cells that line the mucosal surfaces inside the head and neck and can arise from the mucosal surfaces of the oral cavity (OSCC), oropharynx (OPSCC), and larynx. Head and neck cancers can also begin in the salivary glands and in paranasal sinuses and nasal cavities ${ }^{41,42}$. We showed that the circRNA circPVT1 acts as an oncogene in head and neck squamous cell carcinoma $(\text { HNSCC })^{39}$. CircPVT1 expression is regulated through the mut-p53/YAP/TEAD complex binding its own promoter, which is independent from the host gene PVT1 promoter $^{39}$. CircPVT1 is overexpressed in tumors compared to matched-non-tumoral tissues and its expression is particularly high in patients with TP53 mutations ${ }^{39}$. This is an example of a circRNA acting as an oncogene and modulating the expression of miR-497-5p and some of its targets, such as aurka, mki67, and bub1, all genes involved in the control of cell proliferation. This is in line with the known role of miR-497-5p as a tumor suppressor in several cancers ${ }^{39,43-46}$.

Using high-throughput sequencing and RT-qPCR, Li et al. ${ }^{47}$ showed that hsa_circ_0008309 is down-regulated in OSCC tissues relative to paired adjacent normal tissues $(\mathrm{ANTs})^{47,48}$ and statistically correlated with pathological differentiation ${ }^{48}$. Intriguingly, bioinformatics analysis showed that hsa_circ_0008309 might function within a molecular network involving miR-1290, miR-136-5p, miR-382-5p and the ATXN1 gene, coding for the DNAbinding protein Ataxin-1 (refs. ${ }^{47,48}$ ).

Xuan et al. $^{8}$ analyzed the circRNA expression in a cohort of Laryngeal squamous cell carcinoma (LSCC) tissues. They found that two circRNAs, hsa_circRNA_100855 and hsa_circRNA_104912, were respectively up- and down-regulated in cancer tissues in comparison to the corresponding adjacent non-neoplastic tissues ${ }^{8}$. Patients with T3-4 stage, neck nodal metastasis, or advanced clinical stage had higher hsa_circRNA_100855 expression and a lower hsa_circRNA_104912 expression $^{8,48}$.

CircHIPK3 is highly expressed in nasopharyngeal carcinoma (NPC) ${ }^{9}$. The silencing of circHIPK3 can reduce cell proliferation, migration, and invasion in vitro and it can repress tumor growth and metastasis in vivo ${ }^{9}$. CircHIPK3 functions in NPC by sponging the miR-4288, which in turn targets the E74-like ETS transcription factor 3 (ELF3) $^{9}$. Studying the circHIPK3-miR-4288-ELF3 molecular pathway could instruct ways to identify new therapeutic strategies focused on this regulatory loop.

\section{CircRNAs in breast cancer}

Breast cancer is the most common cancer in females and it can be classified in three major cancer subtypes according to estrogen or progesterone receptor expression and $E R B B 2$ gene amplification: hormone receptor positive/ERBB2 negative (HR+/ERBB2-), ERBB2 positive $(\mathrm{ERBB} 2+)$, and triple-negative ${ }^{49}$.

Galasso et al. performed a pilot study in which they described one of the first panels of circRNAs expressed in breast cancer by analyzing RNA sequencing data from five paired breast cancer samples ${ }^{50}$. At the same time, Nair et $\mathrm{al} .{ }^{51}$ developed an automated workflow called Circ-Seq to identify circRNAs in breast tumors and breast cancer cell lines. A recent work identified 235 differentially expressed circRNAs in breast cancer through highthroughput circRNA microarray analysis ${ }^{52}$. Among all the modulated circRNAs, circTADA2A-E6 (hsa_circ_0006220) and circTADA2A-E5/E6 (hsa_circ_0043278) were ranked in the top five down-regulated circRNAs by microarray analysis ${ }^{52}$. In particular, circTADA2A-E6 sponges miR-203a-3p and functions as a tumor suppressor by inhibiting cell proliferation, migration, and metastasis. The SOCS3 gene was predicted as a downstream target gene of the circTADA2A-E6/miR$203 a-3 p a^{2} s^{52}$, and a previous study reported that miR203a-3p promotes cell proliferation by targeting SOCS3 in MCF-7 cells ${ }^{53}$. These results show that the circTADA2AE6/miR-203a-3p/SOCS3 axis plays an important role in the inhibition of breast cancer progression.

CircRNA-000911 is another circRNA acting in breast cancer as a tumor suppressor ${ }^{54}$. Wang et al. ${ }^{54}$ showed that 
circRNA-000911 suppresses the proliferative, migratory, and invasive capacities of breast cancer cells by sponging miR-449a and releasing Notch1, a functional target of miR449a. This mechanism includes the involvement of Ago2, an essential protein for circRNA sponge activity ${ }^{4,55}$. The consequence of circRNA-000911 down-regulation in breast cancer is the up-regulation of miR-449a and downregulation of Notch1. Importantly, one downstream effector of Notch1 is the nuclear factor-kB (kB), which normally promotes breast cancer tumorigenesis and progression ${ }^{54}$.

The circRNA circEPSTI1 (hsa_ circRNA_000479) is upregulated in breast cancer and it is a prognostic marker and mediator of triple-negative breast cancer (TNBC) progression ${ }^{56}$. CircEPSTI1 behaves as an oncogene promoting TNBC cell proliferation in vitro and in vivo, and it is able to sponge both miR-4753 and miR-6809 (ref. ${ }^{56}$ ). BCL11A is a direct target gene of both miRNAs, and it is inhibited as a consequence of circEPSTI1 knockdown. It follows that the circEPSTI1-miR-4753/6809-BCL11A axis could be an interesting pathway to investigate in order to identify new therapeutic strategies for the treatment of $\mathrm{TNBC}^{56}$.

CircANKS1B is another circRNA up-regulated in TNBC and its expression is associated with both lymph node metastasis and advanced clinical stage ${ }^{57}$. CircANKS1B is able to sponge miR-148a-3p and miR-152$3 p$, thereby increases the expression of transcription factor USF1, which in turn up-regulates TGF- $\beta 1$ expres$\operatorname{sion}^{57}$. The up-regulation of TGF- $\beta 1$ results in activation of the TGF- $\beta 1 /$ Smad signaling pathway, promoting epithelial-to-mesenchymal transition $(\mathrm{EMT})^{57}$. The results suggest that circANKS1B is an interesting circRNAs to study in order to find alternative therapeutic strategies for inhibiting breast cancer metastasis.

\section{CircRNAs in lung cancer}

Lung cancer is one of the most common cancers in the world with 5 -year survival rates varying from 92 to $0 \%$, depending on disease stage at diagnosis ${ }^{58}$.

Circ-ITCH is generated from several exons of the ITCH E3 ubiquitin protein ligase (ITCH) and it shares the miR-7 and miR-214 binding sites with the three-prime untranslated regions ( $3^{\prime}$-UTR) of its parental gene $\mathrm{ITCH}^{59}$. Circ-ITCH plays an inhibitory role in lung cancer progression by sponging miR-7 and miR-214 and regulating the expression of $\mathrm{ITCH}^{59,60}$. ITCH negatively regulates canonical Wnt signaling by targeting the dishevelled-2 (Dvl2) protein ${ }^{61}$. In lung cancer the downregulation of circ-ITCH brings to an increase of miR-7 and miR-214, thereby to a decrease of their target gene, ITCH. As a consequence, the Wnt/ $\beta$-catenin pathway is enhanced, thereby promoting the development and progression of cancer ${ }^{59,60}$. Another circRNA that indirectly affects ITCH expression is hsa_circ_0043256. The
circRNA hsa_circ_0043256 is able to sponge miR-1252, which binds the ITCH $3^{\prime}-\mathrm{UTR}^{62}$. Both circRNAs, circITCH and hsa-circ_0043256, behave as tumor suppressors in lung cancer and their combined action could be used to design new strategies for the treatment of this malignancy.

In contrast to circ-ITCH, hsa_circ_0012673 is overexpressed in lung adenocarcinoma and promotes cell proliferation through the miR-22/ErbB3 pathway ${ }^{63}$. Hsa_circ_0012673 is able to sponge miR-22, which targets ERBB3/HER3, an important receptor tyrosine kinase in lung adenocarcinoma. ERBB3/HER3 is a member of the epidermal growth factor receptor (EGFR/ERBB) family ${ }^{64}$ and EGFR mutations were characterized for their important role in lung cancer ${ }^{65}$.

\section{CircRNA in Alzheimer's disease}

Alzheimer's disease (AD) is the most prevalent cause of dementia affecting millions of people worldwide ${ }^{66}$. AD is a progressive and neurodegenerative disorder characterized by widespread neuronal atrophy and two histopathological hallmarks: extracellular senile plaques consisting of amyloid- $\beta$ peptides, and intracellular neurofibrillary tangles composed of abnormally hyperphosphorylated Tau protein ${ }^{66}$.

Dube et al. ${ }^{67}$ generated RNA-seq data from individuals with and without $\mathrm{AD}$ to quantify cortical circRNA expression. The results showed that there are significant associations between circRNA expression and AD diagnosis, clinical dementia severity, and neuropathological severity ${ }^{67}$. Interestingly, circRNA expression changes can be observed early on, in pre-symptomatic $\mathrm{AD}$ and in autosomal dominant $\mathrm{AD}^{67}$. The microtubule-associated Tau protein plays a central role in $\mathrm{AD}$ since it is responsible for amyloid-beta induced neuronal cell death $^{68}$. The MAPT gene generates the Tau protein. Using a PCR screen of RNA from human brain tissues, Welden et al. showed that the MAPT locus generates circRNAs through a backsplicing mechanism, but the role of these circRNAs is still unclear ${ }^{69}$.

Similarly, CDR1as has been one of the first circRNAs that were characterized. It derives from the cerebellar degeneration-related protein 1 antisense transcript (CDR1AS) and contains over 70 conventional binding sites for miR- $7^{4,33,70}$. Down-regulation of CDR1as causes up-regulation of miR-7 and, consequently, negative regulation of its respective targets, such as ubiquitin protein ligase A (UBE2A) $)^{71-73}$. UBE2A is important for clearing amyloid peptides and it was found depleted in the $A D$ brain $^{71-73}$.

\section{CircRNAs in cardiovascular diseases}

RNA-Seq analysis of ribosome-depleted libraries from hearts of human, mouse, and rats origins, detected more 
than 9000 candidate circRNAs for each species ${ }^{74}$. A similar analysis listed more than 15,000 cardiac circRNAs in humans ${ }^{75}$. Although the study showed no statistically significant circRNA that was differentially expressed in diseased hearts compared to healthy hearts, other studies are needed to elucidate the role of circRNAs in cardiac diseases $^{75}$. On the other hand, the analysis found significant differential expressed circRNAs during cardiomyocyte differentiation ${ }^{75}$.

Many of the identified cardiac circRNAs are yet uncharacterized in terms of their specific function. Nevertheless, the identification of cardiac circRNAs represents a potential strategy to use circRNAs as target molecules in the prevention and treatment of cardiovascular diseases.

The first circRNA described with a cardioprotective role was the heart-related circRNA, HRCR. This circRNA acts as a miR-223 sponge to inhibit cardiac hypertrophy and heart failure ${ }^{76}$. MiR-223 is able to suppress the expression level of its target, ARC, the apoptosis repressor with CARD domain protein. HRCR acts as an antihypertrophic molecule due to its sponging mechanism toward miR-223, which causes up-regulation of $\mathrm{ARC}^{76}$.

More recently, circFndc3b was identified as another circRNA involved in cardioprotection. CircFndc3b interacts with the RNA-binding protein Fused in Sarcoma (FUS) to regulate VEGF expression and signaling ${ }^{12}$. Acting on the FUS/VEGF-A axis, circFBDc3b is able to enhance angiogenesis and retard cardiomyocytes and endothelial cell apoptosis ${ }^{12}$.

Yet another circRNA, Cdrlas (ciRS-7), acts as a miR-7a sponge in myocardial cells ${ }^{77}$. It was shown that ciRS-7 induces apoptosis in myocardial infarction (MI) in mice by means of increasing caspase- 3 activity. CiRS-7 is upregulated in infarcted hearts, and it is able to inhibit the miR-7a mediated cardiomyocyte protection against MI injury acting as a miRNA sponge ${ }^{77,78}$. CiRS-7's sponge mechanism toward miR-7a determines the up-regulation of two miR-7a targets, PARP and SP1. These proteins play pro-apoptotic roles during $\mathrm{MI}^{77}$.

MFACR (mitochondrial fission and apoptosis-related circRNA) regulates mitochondrial fission and apoptosis in the heart, while acting as a miRNA sponge for miR-652$3 \mathrm{p}^{79}$. MiR-652-3p down-regulates its target, MTP18, a nuclear-encoded mitochondrial membrane protein that contributes to mitochondrial fission in mammalian cells $^{79,80}$. As a result, the MFACR-activated pathway instigates cardiomyocyte death through miR-652-3pdependent up-regulation of MTP18 expression ${ }^{79}$.

Another circRNA involved in cardiomyocyte apoptotic events is circNCX1, which is generated from the sodium/ calcium exchanger 1 (ncx1) gene ${ }^{81}$. circNCX1 acts as a miRNA sponge for miR-133a-3p, which is able to target the pro-apoptotic gene called Cell Death-Inducing p53- target Protein 1 (CDIP1). Importantly, miR-133a-3p plays a cardioprotective role and it is suppressed by the circNCX1 sponge mechanism ${ }^{81}$. This is an example of circRNAs that enhances damage following a MI event, primarily by promoting apoptosis of cardiomyocytes ${ }^{81}$.

\section{CircRNAs in diabetes}

Diabetes is a group of metabolic disorders all characterized by hyperglycemia, namely high levels of sugar in the blood. This condition is associated with various pathological states, such as cardiovascular disease, retinopathy, nephropathy, and neuropathy ${ }^{82}$.

A recent work showed the human pancreatic islets express thousands of circRNAs ${ }^{83}$. The circRNA Cdr1as is already known for its miR-7 sponging activity in embryonic zebrafish brains and in infarcted hearts ${ }^{4,34,84}$. Moreover, Cdr1as is able to affect miR-7 function in adult islet cells ${ }^{84}$. Xu et al. $^{84}$ showed that miR-7 is highly expressed in islet cells, and its overexpression in transgenic mice $\beta$-cells causes diabetes due to impaired insulin secretion and $\beta$ cell dedifferentiation. Cdr1as promotes insulin secretion by sponging miR-7 in islet cells ${ }^{84}$. Hence, the interaction between Cdrlas and miR-7 in insulin secretion may become a new therapeutic target for improving $\beta$ cell function in diabetes ${ }^{84}$.

The circRNA circHIPK3 was found up-regulated in retinas and retinal endothelial cells of patients with diabetes. CircHIPK3 is able to regulate the retinal vascular endothelial function while sponging miR-30a-3p ${ }^{85}$. As a consequence of its action as miRNA sponge, circHIPK3 increases the expression of VEGFC, FZD4, and WNT2, leading to endothelial proliferation and vascular dysfunction ${ }^{85}$. It follows that circHIPK3 could serve as a valid target for diabetic retinopathy.

\section{CircRNAs as potential disease biomarkers}

Both prognostic biomarkers and markers that predict responses to a drug or other treatment modalities must bear high specificity for a given pathophysiological condition and a highly reproducible detection capacity. Accordingly, the renewed identification of circRNAs has opened a new potential strategy for diagnosis and for monitoring progression of different human diseases (Table 1). This is primarily due to the high tissue specificity of circRNAs, their relatively high stability in tissues and body fluids, as well as ease of detection using rather simple technologies, such as real-time $\mathrm{PCR}^{1,86,87}$. CircRNAs are highly abundant in blood ${ }^{25}$ and there are also evidences of circRNAs in urine samples ${ }^{26}$, for example to assist monitoring of patients who have undergone kidney transplantation, or for diagnosis of patients with bladder cancer $^{26,88}$.

Importantly, several recent studies reported the presence of circRNAs in extracellular vesicles, mainly 
Table 1 CircRNAs as potential biomarkers of human diseases.

\begin{tabular}{|c|c|c|c|}
\hline CircRNA & $\begin{array}{l}\text { Disease/ } \\
\text { expression }\end{array}$ & Detection method & Refs. \\
\hline hsa_circ_0000190 & GC/decreased & qRT-PCR & 96 \\
\hline hsa_circ_002059 & GC/decreased & qRT-PCR & 97 \\
\hline circFARSA & NSCLC/increased & RNA-seq; qRT-PCR & 98 \\
\hline F-circEA-2a & NSCLC/increased & qRT-PCR & 99 \\
\hline hsa_circ_0027089 & LIHC/Increased & Microarray; qRT-PCR & 100 \\
\hline circ-LPAR1 & $A D /$ increased & Microarray; qRT-PCR & 102 \\
\hline circ-AXL & AD/increased & Microarray; qRT-PCR & 102 \\
\hline circ-GPHN & AD/increased & Microarray; qRT-PCR & 102 \\
\hline circ-PCCA & AD/decreased & Microarray; qRT-PCR & 102 \\
\hline circ-HAUS4 & AD/decreased & Microarray; qRT-PCR & 102 \\
\hline circ-KIF18B & AD/decreased & Microarray; qRT-PCR & 102 \\
\hline circ-ТТС39C & AD/decreased & Microarray; qRT-PCR & 102 \\
\hline hsa_circRNA_405619 & AD/increased & Microarray; qRT-PCR & 103 \\
\hline hsa_circRNA_000843 & AD/increased & Microarray; qRT-PCR & 103 \\
\hline hsa_circRNA_100861 & AD/decreased & Microarray; qRT-PCR & 103 \\
\hline hsa_circRNA_102448 & AD/decreased & Microarray; qRT-PCR & 103 \\
\hline hsa_circRNA_025016 & PAF/increased & Microarray; qRT-PCR & 106,107 \\
\hline MICRA & LVD/increased & qRT-PCR & 106,107 \\
\hline CircANRIL & ATH/Increased & qRT-PCR & 108 \\
\hline
\end{tabular}

Listed are examples of circRNAs that might serve as biomarkers of various diseases.

GC gastric cancer, NSCLC non-small cell lung cancer, LIHC liver hepatocellular carcinoma, AD Alzheimer's disease, PAF postoperative atrial fibrillation, LVD: left ventricular dysfunction, $A T H$ atherosclerosis.

exosomes, which are targets for discovery of additional types of new biomarkers ${ }^{8-91}$. The abundance and diversity of circRNAs in human blood exosomes is already available in a database called exoRBase ${ }^{92}$. Likewise, circRNAs with diagnostic potential have been found in urine exosomes ${ }^{93,94}$. Additionally, another database, MiOncoCirc, was created based on sequencing of more than 2000 tumor samples, and many urine circRNAs were identified as possible biomarkers for prostate cancer ${ }^{95}$.

There are several papers that have shown correlations between expression of specific circRNAs and tumor grade, size, metastatic spread, and lymph node involvement. This is the case of hsa_circ_002059 and hsa_circ_0000190, which were found to be decreased in plasma of patients with gastric cancer ${ }^{96,97}$. Likewise, it has been reported that circFARSA is elevated in plasma of patients with non-small-cell lung cancer (NSCLC), in direct association with tumor cell aggressiveness in vitro ${ }^{98}$. The circRNA F-circEA-2a is another candidate biomarker in NSCLC. Generated from the prevalent fusion gene in lung cancer, $E M L 4-A L K$, circRNA FcircEA-2a appears elevated in plasma samples ${ }^{99}$.

A screening seeking differentially expressed circRNAs in plasma of patients with hepatocellular carcinoma related to the hepatitis B virus, reported elevated expression of hsa_circ_0027089 and classified it as a potential biomarker ${ }^{100}$. Additionally, an atlas of BloodBased Biomarkers for Early Diagnosis of Cancers (BBcancer) has recently been established ${ }^{101}$. It includes data from 5000 samples across 15 different types of cancer $^{101}$.

A recent study evaluated the presence of circRNAs in cerebrospinal fluid of patients with Alzheimer's disease (AD) and found 112 up-regulated and 51 down-regulated circRNAs $^{102}$. Some of these circRNAs were confirmed by real-time PCR, with circ-LPAR1, circ-AXL, and circGPHN elevated and circ-PCCA, circ-HAUS4, circKIF18B, and circ-TTC39C decreased in patients with $\mathrm{AD}^{102}$.

Another study showed that it is possible to differentiate patients with $\mathrm{AD}$ and healthy individuals by testing the expression of circRNAs in peripheral blood mononuclear cells $(\mathrm{PMBCs})^{103}$. Hsa_circRNA_405619 and hsa_circRNA_000843 were shown to be elevated in PBMCs of patients with $\mathrm{AD}$ in comparison to healthy individuals, while hsa_circRNA_100861 and hsa_circRNA_102448 appear decreased in the same patients ${ }^{103}$.

Several published reports relate to circRNAs in different cardiovascular diseases ${ }^{104,105}$. The presence of hsa_circRNA_025016 in the plasma of patients is able to predict postoperative atrial fibrillation, while MICRA (myocardial infarction-associated circRNA) can help predicting left ventricular dysfunction in patients with acute myocardial infarction ${ }^{106,107}$. Similarly, in addition to being much more expressed than its linear form, an isoform of circANRIL has been shown to be elevated in whole blood of cardiac patients and playing an atheroprotective role, unlike its linear counterpart, which appears to play a proatherogenic role ${ }^{108}$.

\section{CircRNAs implicated in molecular pathways disclose their potential use as therapeutic molecules}

Although several circRNAs have been found to be either up- or down-regulated in human tissues, not always their specific role in molecular pathways has been elucidated. Specific circRNAs act in the Wnt signal transduction pathway: circRNA ITCH is active in lung cancer ${ }^{59}$ and cZNF292 is active in glioma ${ }^{109}$ (Fig. 2). Silencing cZNF292 blocked glioma cell cycle progression by means of inhibiting the Wnt/B-catenin signaling pathway ${ }^{109}$.

Wnt signaling can be divided into $\beta$-catenin-dependent (or canonical) and $\beta$-catenin-independent (or non-canonical) signaling $22,110,111$. This pathway plays a critical role 


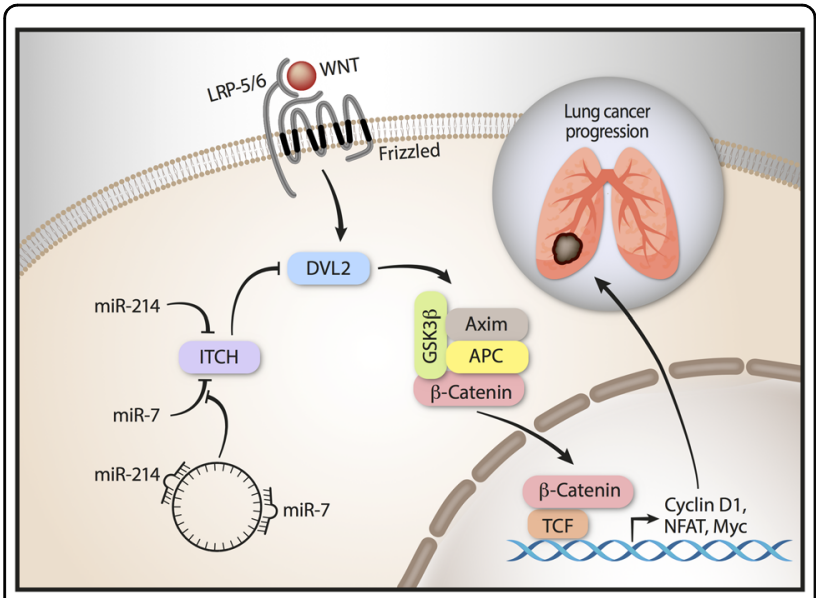

Fig. 2 circ-ITCH working in the WNT signaling pathway. Circ-ITCH negatively regulates the canonical Wnt signaling in lung cancer by sponging miR-7 and miR-214 and regulating the expression of ITCH.

during embryonic development, including cell fate specification, cell proliferation, and cell migration. Moreover, the role of Wnt signaling has been well characterized in several diseases, such as cancer, diabetes, and cardiovascular disorders ${ }^{112-114}$. Accordingly, clinical trials that tested Wnt signaling drugs have shown promising outcomes, and circRNAs affecting the Wnt pathway might serve as targets for new therapies ${ }^{22,115}$.

CircANKS1B promotes the epithelial-to-mesenchymal transition (EMT) in triple-negative breast cancer $(\mathrm{TNBC})^{57}$ (Fig. 3). EMT takes place in a diverse range of physiological and pathological conditions ${ }^{116}$. The molecular reprogramming occurring during EMT is orchestrated by a complex combination of factors, possibly including circRNAs. The biogenesis of numerous circRNAs is promoted during EMT transition by the RNAbinding protein quaking-5 (QKI-5 $)^{16}$. Recently, several clinical trials have been launched based on the current knowledge of EMT heterogeneity and plasticity ${ }^{117}$. The next challenge will be to include circRNAs as biomarkers or pharmacological targets in the protocols of new clinical trials addressing EMT.

The circPVT1 and the circPPP1R12A act within the Hippo-YAP signaling pathway, respectively in head and neck squamous cell carcinoma and in colon cancer ${ }^{19,39}$ (Fig. 4). The Hippo pathway is recognized as an evolutionarily conserved signal transduction pathway that controls proliferation, organ size, and shape during development ${ }^{23}$. Moreover, the Hippo pathway is involved in multiple physiological processes, such as tissue growth, regeneration, and repair, maintaining the tissue homeostasis $^{23}$. Hippo signaling plays an important role as a tumor suppressor in cancer and its deregulation is a key feature for cancer development, progression, and resistance to cancer treatment ${ }^{23,118,119}$. We showed that the

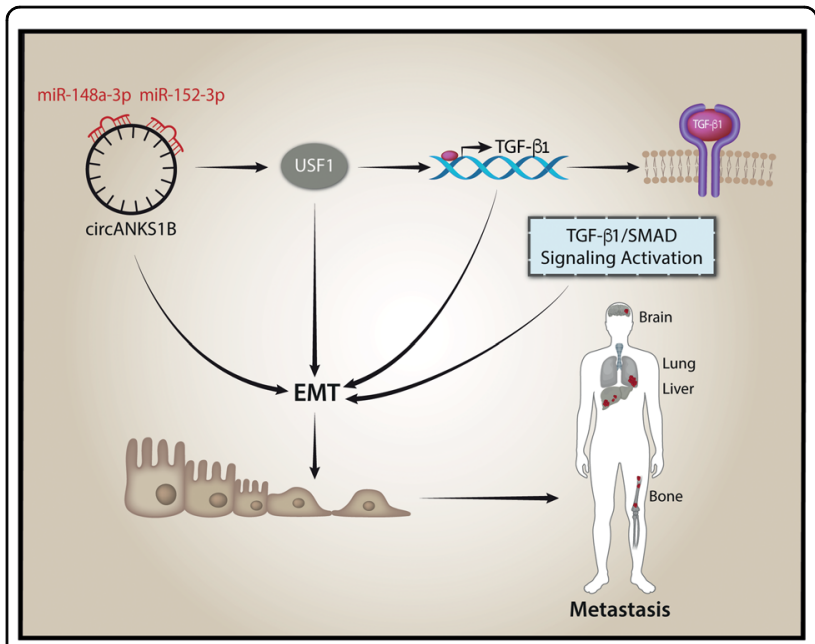

Fig. 3 circANKS1B working in the TGF- $\beta 1$ signaling pathway. CircANKS1B promotes the epithelial-to-mesenchymal transition (EMT) in triple-negative breast cancer sponging the miR-148a-3p and miR152-3p, enhancing the expression of transcription factor USF1 and activating the TGF- $\beta 1 /$ Smad signaling pathway.

mutant form of p53 (mut-p53) physically interacts with the transcriptional cofactor Yes-Associated Protein (YAP) in breast cancer ${ }^{120}$. YAP and TAZ are the main effectors of the Hippo pathway ${ }^{120}$. Hippo pathway inactivation determines the translocation to the nucleus of YAP and TAZ that regulate transcriptional activation in collaboration with mut-p53. In this context, Hippo effectors YAP and TAZ can act either as tumor suppressors, when located in the cytoplasm, or as oncogenes in the nucleus. In its wild type conformation, p53 works as a tumor suppressor regulating the cellular homeostasis ${ }^{23}$. At the same time the "p53 status", wild type or mutant, can be considered a critical point in determining the tumor suppressor or oncogenic activity of the Hippo pathway ${ }^{23}$. Currently, there are several pathway modulators of the Hippo pathway that are subject of clinical development, such as the Verterpofin who inhibits the YAP-TEAD interaction $^{121}$ or the PRIMA1-MET that restores the proapoptotic function of p53 with consequent activation of downstream target genes ${ }^{122}$. Importantly, we showed that YAP binds circPVT1 in head and neck squamous cell carcinoma. Moreover, we demonstrated that mut-p53 stabilizes the YAP/circPVT1 complex ${ }^{39}$. Thus, the current knowledge of circRNAs and their interaction with the Hippo pathway are expected to open new ways for the development of novel and more effective drugs.

\section{Divergent views of circRNA biogenesis and their mode of action}

Despite the great interest that the circRNAs are raising in the scientific community, there are still some important questions regarding their biogenesis and function. The 


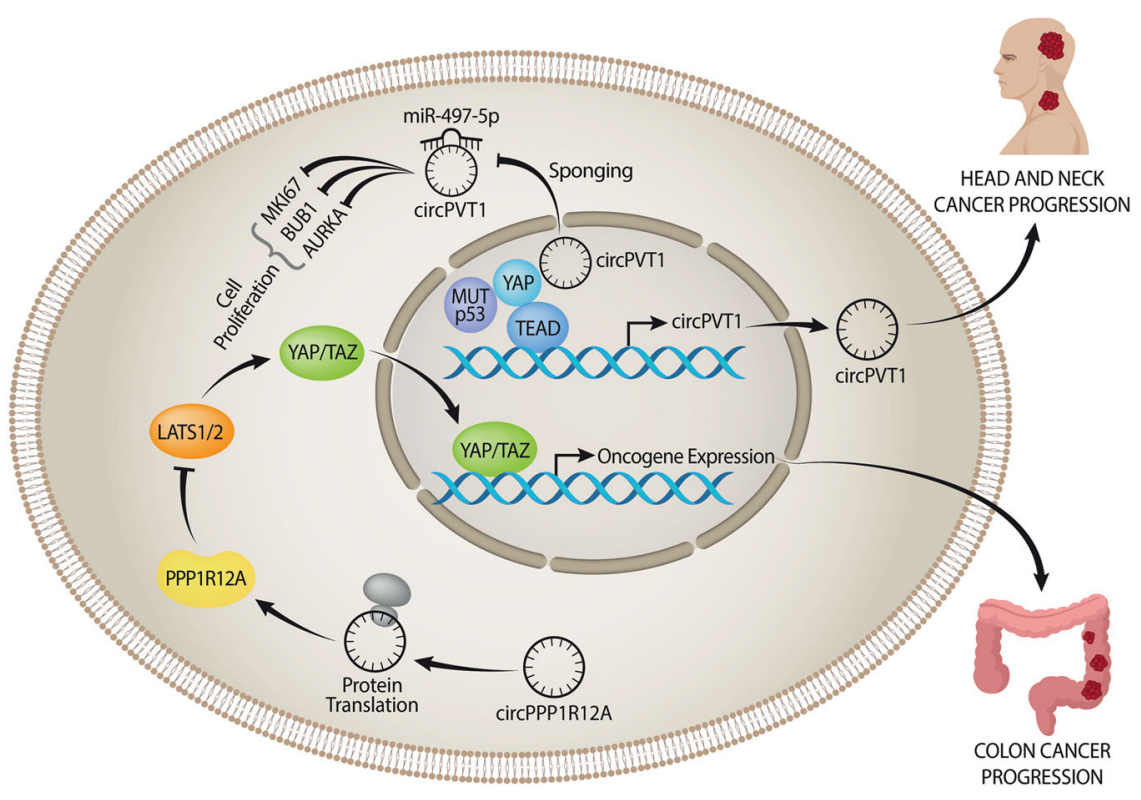

Fig. 4 circPVT1 and circPPP1R12A working in the Hippo signaling pathway. The circPVT1 acts as oncogene in head and neck squamous cell carcinoma sponging the miR-497-5p and binding the complex YAP/mutp53. The circPPP1R12A can be translated into a protein and acts as oncogene in colon cancer where it is able to activated the proliferation of the tumor cells via theHippo-YAP pathway.

presence of repetitive inverted Alu elements flanking exons favors RNA circularization ${ }^{123}$. However, Zhang et al. demonstrated another mechanism not dependent on repetitive sequences for the generation of circRNAs, and occurring by the pairing between complementary sequences in introns flanking exons ${ }^{32}$. Another regulatory mechanism for circRNA biogenesis uses the ADAR protein, which is capable of modifying nucleotides in intronic repeat sequences $^{29}$. Ivanov et al. $^{29}$ demonstrated that ADAR antagonizes the expression of several circRNAs by editing intronic sites that flank exons and promote back-splicing. It is becoming clearer that introns are more important sequences for circRNAs biogenesis than initially anticipated, and that specific proteins might regulate backsplicing. Apparently, several different mechanisms control circRNA biogenesis, but it is yet unclear how do they work and which one, if any, is predominant over the others.

The unique circular configuration confers to cirRNAs not only resistance to digestion by ribonucleases, but it also translates to a longer half-life compared to the respective mRNAs ${ }^{124-126}$. As a consequence, circRNA levels are typically reduced in rapidly proliferating cells, such as in cancer cells. Thus, the association between lower circRNA levels and cancer could be due, in some cases, to a simple dilution effect mediated by cell division, as in colorectal cancer ${ }^{127}$. Hence, the meaning of circRNA expression levels should be carefully evaluated based on the specific cellular context. In addition, many circRNAs are sensitive to RNase $\mathrm{R}$ treatments, thus contradicting claims related to high stability of these molecules ${ }^{128}$.
Although the microRNA sponging mechanism is the better-described role for circRNAs, many circRNAs putatively act as sponges toward only a single, or very few miRNA targets ${ }^{129}$. Notably, there are some prerequisites that should be fulfilled in order to identify a circRNA as a putative ceRNA: the presence of multiple binding sites for the miRNA target, relatively high abundance of the circRNA, a miRNA target with fewer target genes, and a circRNA with a better affinity toward the miRNA than the mRNAs-miRNA affinity ${ }^{130}$. Moreover, a circRNA that triggers the degradation process of a target miRNA, and not only inhibits the interaction between miRNA and mRNA, might act as a better ceRNA candidate ${ }^{129}$.

The most common approach for assessing the sponging mechanism is the ectopic overexpression of binding sites for a specific miRNA. However, the result of this kind of experiment should be interpreted with caution since it could be biased by the introduction of sufficiently high numbers of binding sites able to inhibit the activity of the miRNA in question ${ }^{126}$. At the same time, one should also keep in mind that if a circRNA only binds with the cognate miRNA and inhibits its function without degrading it, the abundance of the miRNA would not be affected. Piwecka et al. ${ }^{131}$ have shown that the choice between either degradation or functional inhibition depends on whether the binding sites connecting circRNAs and miRNAs are completely complementary or they only partially match each other. It follows that a reliable in silico analysis of putative binding sites for circRNAs on miRNA targets is an essential requirement. While most 
studies have shown repression of miRNAs, Hansen et al. ${ }^{132}$ showed that the interaction between $\mathrm{Cdr} 1$ and miR-671 would actually lead to degradation of this circRNA through AGO2 rather than by the expected miRNA degradation mode ${ }^{132}$. Therefore, it is possible that additional circRNA-miRNA interactions regulate RNA circles.

With a few exceptions, the majority of circRNAs are expressed at low levels in both normal and cancer cells; hence they are unlikely to have only secondary roles in cellular physiology. However, the cascade of events that a single circRNA might unleash can potentially be of great importance from the clinical point of view, as we have shown for the circRNA circPVT1 (ref. ${ }^{39}$ ). The roles of circRNAs must be carefully assessed in light of the various processes of their biogenesis and degradation, in addition to their broad capabilities for interacting with miRNAs and proteins.

\section{Conclusions}

The biochemical and molecular characteristics of circRNAs hold the promise that specific circles of RNA will be utilized in the future as disease biomarkers and pharmacological targets, thus opening new possibilities for early detection and treatment ${ }^{133-135}$. The large spectrum of mechanisms of action used by circRNAs makes the understanding of their role not only challenging but also promising in terms of resolving the complex molecular mechanisms activated in human disorders. Indeed, circRNAs can act as tumor suppressors or as oncogenes in oncology ${ }^{136,137}$. Likewise, circRNAs are involved in cardioprotection against heart failure, as well as mediate cardiomyocyte death in myocardial infarction ${ }^{12,76-79}$. Moreover, circRNAs are extensively expressed in the mammalian brain ${ }^{138,139}$. Networks of circRNAs, RNAbinding proteins and microRNAs play important roles in different human diseases, which reflects the complex regulatory potential of circRNAs. Hence, it is likely that the next few years will witness the discovery of more circRNAs and new modes of their action in human disorders.

\section{Acknowledgements}

We would like to thank the support of AIRC, Lazio Innova/Regione Lazio, MAECI Italy/USA bilateral grant program, and Alliance Against Cancer (ACC). Moreover, we would like to thank Professor Yosef Yarden's laboratory for its kind collaboration. Y.Y. is the incumbent of the Harold and Zelda Goldenberg Professorial Chair.

\footnotetext{
Author details

'Unit of Oncogenomic and Epigenetic, Department of Research, Advanced Diagnostic, and Technological Innovation, IRCCS, Regina Elena National Cancer Institute, Rome, Italy. ${ }^{2}$ Department of Biological Regulation, Weizmann Institute of Science, Rehovot, Israel. ${ }^{3}$ SAFU Unit, Department of Research, Advanced Diagnostic, and Technological Innovation, IRCCS, Regina Elena National Cancer Institute, Rome, Italy
}

\section{Author contributions}

Guarantors of the article: G.B. and Y.Y.; L.V. and G.B. identified the scope of this review article and wrote and revised the review. Y.Y. and E.T. revised the review and contributed to the writing. S.S. revised the review. L.V. conceived the original figures. E.T. revised the figures. All authors approved the final manuscript and agreed to be responsible for this review.

\section{Funding}

This work was supported by AIRC IG 2017 ID 20613 and fondazione AIRC under 5 per mille 2019 - ID. 22759 program - GL Blandino Giovanni. Lazio Innova/ Regione Lazio, MAECI Italy/USA bilateral grant program, and Alliance Against Cancer (ACC). YY laboratory was supported by the European Research Council, the Israel Science Foundation, the Israel Cancer Research Fund, and the Dr. Miriam and Sheldon G. Adelson Medical Research Foundation.

\section{Ethics}

This study did not require ethical approval.

\section{Conflict of interest}

The authors declare no competing interests.

\section{Publisher's note}

Springer Nature remains neutral with regard to jurisdictional claims in published maps and institutional affiliations.

Received: 20 October 2020 Revised: 18 April 2021 Accepted: 19 April 2021 Published online: 11 May 2021

\section{References}

1. Jeck, W. R. \& Sharpless, N. E. Detecting and characterizing circular RNAs. Nat. Biotechnol. 32, 453-461 (2014).

2. Verduci, L., Strano, S., Yarden, Y. \& Blandino, G. The circRNA-microRNA code: emerging implications for cancer diagnosis and treatment. Mol. Oncol. 13, 669-680 (2019).

3. Rong, D. et al. An emerging function of circRNA-miRNAs-mRNA axis in human diseases. Oncotarget 8, 73271-73281 (2017).

4. Memczak, $\mathrm{S}$. et al. Circular RNAs are a large class of animal RNAs with regulatory potency. Nature 495, 333-338 (2013).

5. Hsiao, K. Y. et al. Noncoding effects of circular RNA CCDC66 promote colon cancer growth and metastasis. Cancer Res. 77, 2339-2350 (2017).

6. Han, D. et al. Circular RNA circMTO1 acts as the sponge of microRNA-9 to suppress hepatocellular carcinoma progression. Hepatology 66, 1151-1164 (2017).

7. Song, T. et al. CircRNA hsa_circRNA_101996 increases cenvical cancer proliferation and invasion through activating TPX2 expression by restraining miR-8075. J. Cell Physiol. 234, 14296-14305 (2019).

8. Xuan, L. et al. Circular RNA: a novel biomarker for progressive laryngeal cancer. Am. J. Transl. Res. 8, 932-939 (2016).

9. Ke, Z., Xie, F., Zheng, C. \& Chen, D. CircHIPK3 promotes proliferation and invasion in nasopharyngeal carcinoma by abrogating miR-4288-induced ELF3 inhibition. J. Cell Physiol. 234, 1699-1706 (2019).

10. Du, W. W. et al. Foxo3 circular RNA retards cell cycle progression via forming ternary complexes with p21 and CDK2. Nucleic Acids Res. 44, 2846-2858 (2016).

11. He, Q. et al. circ-SHKBP1 regulates the angiogenesis of U87 glioma-exposed endothelial cells through miR-544a/FOXP1 and miR-379/FOXP2 pathways. Mol. Ther. Nucleic Acids 10, 331-348 (2018).

12. Garikipati, V. N. S. et al. Circular RNA CircFndc3b modulates cardiac repair after myocardial infarction via FUSNEGF-A axis. Nat. Commun. 10, 4317 (2019).

13. Geng, H. H. et al. The circular RNA Cdr1as promotes myocardial infarction by mediating the regulation of miR-7a on its target genes expression. PLOS ONE 11, e0151753 (2016).

14. Abdelmohsen, $\mathrm{K}$. et al. Identification of HuR target circular RNAs uncovers suppression of PABPN1 translation by CircPABPN1. RNA Biol. 14, 361-369 (2017).

15. Yang, Q. et al. A circular RNA promotes tumorigenesis by inducing c-myc nuclear translocation. Cell Death Differ. 24, 1609-1620 (2017). 
16. Conn, S. J. et al. The RNA binding protein quaking regulates formation of circRNAs. Cell 160, 1125-1134 (2015).

17. Legnini, I. et al. Circ-ZNF609 is a circular ma that can be translated and functions in myogenesis. Mol. Cell 66, 22-37 (2017).

18. Zhang, M. et al. A novel protein encoded by the circular form of the SHPRH gene suppresses glioma tumorigenesis. Oncogene 37, 1805-1814 (2018).

19. Zheng, X. et al. A novel protein encoded by a circular RNA circPPP1R12A promotes tumor pathogenesis and metastasis of colon cancer via HippoYAP signaling. Mol. Cancer 18, 47 (2019).

20. Li, Z. et al. Exon-intron circular RNAs regulate transcription in the nucleus. Nat. Struct. Mol. Biol. 22, 256-264 (2015).

21. Zhang, Y. et al. Circular intronic long non coding RNAs. Mol. Cell 51, 792-806 (2013).

22. Jung, Y. S. \& Park, J. I. Wnt signaling in cancer: therapeutic targeting of Wnt signaling beyond $\beta$-catenin and the destruction complex. Exp. Mol. Med. $\mathbf{5 2}$ 183-191 (2020).

23. Ferraiuolo, M., Verduci, L., Blandino, G. \& Strano, S. Mutant p53 protein and the hippo transducers YAP and TAZ: a critical oncogenic node in human cancers. Int. J. Mol. Sci. 18, 961 (2017).

24. Bahn, J. H. et al. The landscape of microRNA, Piwi-interacting RNA, and circular RNA in human saliva. Clin. Chem. 61, 221-230 (2015).

25. Memczak, S., Papavasileiou, P., Peters, O. \& Rajewsky, N. Identification and characterization of circular RNAs as a new class of putative biomarkers in human blood. PLoS ONE 10, e0141214 (2015).

26. Jacky Lam, W. K. \& Dennis, Lo. Y. M. Circular RNAs as urinary biomarkers. Clin. Chem. 65, 1196-1198 (2019).

27. Salzman, J., Gawad, C., Wang, P. L., Lacayo, N. \& Brown, P. O. Circular RNAs are the predominant transcript isoform from hundreds of human genes in diverse cell types. PLoS ONE 7, e30733 (2012).

28. Ashwal-Fluss, R. et al. circRNA biogenesis competes with pre-mRNA splicing. Mol. Cell 56, 55-56 (2014).

29. Ivanov, A. et al. Analysis of intron sequences reveals hallmarks of circular RNA biogenesis in animals. Cell Rep. 10, 170-177 (2015).

30. Liang, D. \& Wilusz, J. E. Short intronic repeat sequences facilitate circular RNA production. Genes Dev. 28, 2233-2247 (2014).

31. Starke, S. et al. Exon circularization requires canonical splice signals. Cell Rep. 10, 103-111 (2015)

32. Zhang, X. O. et al. Complementary sequence-mediated exon circularization. Cell 159, 134-147 (2014).

33. Hansen, T. B. et al. Natural RNA circles function as efficient microRNA sponges. Nature 495, 384-388 (2013).

34. $\mathrm{Yu}$, L. et al. The circular RNA Cdr1as act as an oncogene in hepatocellular carcinoma through targeting miR-7 expression. PLOS ONE 11, e0158347 (2016).

35. Li, F. et al. Circular RNA ITCH has inhibitory effect on ESCC by suppressing the Wnt/b-catenin pathway. Oncotarget 6, 6001-6013 (2015).

36. Zheng, Q. et al. Huang, circular RNA profiling reveals an abundant circHIPK3 that regulates cell growth by sponging multiple miRNAs. Nat. Commun. 7, 11215 (2016).

37. Xu, X. W. et al. Circular RNA hsa_circ_000984 promotes colon cancer growth and metastasis by sponging miR-106b. Oncotarget 8 , 91674-91683 (2017).

38. Zheng, J. et al. TTBK2 circular RNA promotes glioma malignancy by regulating miR-217/HNF1b/Derlin-1 pathway. J. Hematol. Oncol. 10, 52 (2017).

39. Verduci, L. et al. The oncogenic role of circPVT1 in head and neck squamous cell carcinoma is mediated through the mutant p53/YAP/TEAD transcriptioncompetent complex. Genome Biol. 18, 237 (2017).

40. Stoll, L. et al. A circular RNA generated from an intron of the insulin gene controls insulin secretion. Nat. Comun. 11, 5611 (2020).

41. Leemans, C. R., Braakhuis, B. J. M. \& Brakenhoff, R. H. The molecular biology of head and neck cancer. Nat. Rev. Cancer 11, 9-22 (2011).

42. O'Rorke, M. A. et al. Human papillomavirus related head and neck cancer survival: a systematic review and meta-analysis. Oral. Oncol. 48, 1191-1201 (2012).

43. Guo, S. T. et al. MicroRNA-497 targets insulin-like growth factor 1 receptor and has a tumour suppressive role in human colorectal cancer. Oncogene $\mathbf{3 2}$ 1910-1920 (2013).

44. Huang, C. et al. MiR-497 suppresses YAP1 and inhibits tumor growth in nonsmall cell lung cancer. Cell Physiol. Biochem. 37, 342-352 (2015).

45. Li, D. et al. Analysis of miR-195 and miR-497 expression, regulation and role in breast cancer. Clin. Cancer Res. 17, 1722-1730 (2011).
46. Wang, S. et al. The potent tumor suppressor miR-497 inhibits cancer phenotypes in nasopharyngeal carcinoma by targeting ANLN and HSPA4L. Oncotarget 6, 35893-35907 (2015).

47. Li, B. et al. Hsa_circ_0008309 may be a potential biomarker for oral squamous cell carcinoma. Dis. Markers 2018, 7496890 (2018).

48. Guo, Y. et al. Circular RNAs and their roles in head and neck cancers. Mol. Cancer 18, 44 (2019).

49. Waks, A. G. \& Winer, E. P. Breast cancer treatment: a review. JAMA 321 288-300 (2019).

50. Galasso, M. et al. Profiling of the predicted circular RNAs in ductal in situ and invasive breast cancer: a pilot study. Int. J. Genomics 2016, 4503840 (2016).

51. Nair, A. A. et al. Circular RNAs and their associations with breast cancer subtypes. Oncotarget 7, 80967-80979 (2016).

52. $\mathrm{Xu}$, J. Z. et al. circTADA2As suppress breast cancer progression and metastasis via targeting miR-203a-3p/SOCS3 axis. Cell Death Dis. 10, 175 (2019).

53. Muhammad, N., Bhattacharya, S., Steele, R. \& Ray, R. B. Anti-miR-203 suppresses ER-positive breast cancer growth and stemness by targeting SOCS3. Oncotarget 7, 58595-58605 (2016).

54. Wang, H., Xiao, Y., Wu, L. \& Ma, D. Comprehensive circular RNA profiling reveals the regulatory role of the circRNA-000911/miR-449a pathway in breast carcinogenesis. Int. J. Oncol. 52, 743-754 (2018).

55. Du, W. W. et al. Identifying and characterizing circRNA-protein interaction. Theranostics 7, 4183-4191 (2017).

56. Chen, B. et al. circEPSTI1 as a prognostic marker and mediator of triplenegative breast cancer progression. Theranostics 8, 4003-4015 (2018).

57. Zeng, K. et al. The pro-metastasis effect of circANKS1B in breast cancer. Mol. Cancer 17, 160 (2018).

58. Neal R. D., Sun F., Emery J. D. \& Callister M. E. Lung Cancer. BMJ 365, 11725 (2019).

59. Wan, L. et al. Circular RNA-TCH suppresses lung cancer proliferation via inhibiting the Wnt/B-catenin pathway. Biomed. Res. Int. 2016, 1579490 (2016).

60. Chen, Y., Wei, S., Wang, X., Zhu, X. \& Han, S. Progress in research on the role of circular RNAs in lung cancer. World J. Surg. Oncol. 16, 215 (2018).

61. Wei, W., Li, M., Wang, J., Nie, F. \& Li, L. The E3 ubiquitin ligase ITCH negatively regulates canonical Wnt signaling by targeting dishevelled protein. Mol.Cell Biol. 32, 3903-3912 (2012).

62. Tian, F., Yu, C. T., Ye, W. D. \& Wang, Q. Cinnamaldehyde induces cell apotosis mediated by a novel circular RNA hsa_circ_0043256 in non-small cell lung cancer. Biochem. Biophys. Res. Commun. 493, 1260-1266 (2017).

63. Wang, X. et al. Increased circular RNA hsa_circ_0012673 acts as a sponge of miR-22 to promote lung adenocarcinoma proliferation. Biochem. Biophys. Res. Commun. 496, 1069-1075 (2018).

64. Kiavue, N. et al. ERBB3 mutations in cancer: biological aspects, prevalence and therapeutics. Oncogene 39, 487-502 (2020).

65. Gazdar, A. F. Activating and resistance mutations of EGFR in non-small-cell lung cancer: role in clinical response to EGFR tyrosine kinase inhibitors. Oncogene 28, S24-S31 (2009).

66. Scheltens, P. et al. Alzheimer's disease. Lancet 388, 505-517 (2016).

67. Dube, $U$. et al. An atlas of cortical circular RNA expression in Alzheimer disease brains demonstrates clinical and pathological associations. Nat. Neurosci. 22, 1903-1912 (2019).

68. Roberson, E. D. et al. Reducing endogenous tau ameliorates amyloid betainduced deficits in an Alzheimer's disease mouse model. Science $\mathbf{3 1 6}$ 750-754 (2007).

69. Welden, J. R., van Doorn, J., Nelson, P. T. \& Stamm, S. The human MAPT locus generates circular RNAs. Biochim. Biophys. Acta Mol. Basis Dis. 1864, 2753-2760 (2018).

70. Zou, Y. et al. The role of circular RNA CDR1as/ciRS-7 in regulating tumor microenvironment: a pan-cancer analysis. Biomolecules 9, 429 (2019).

71. Lukiw, W. J. Variability in micro RNA (miRNA) abundance, speciation and complexity amongst different human populations and potential relevance to Alzheimer's disease (AD). Front. Cell Neurosci. 7, 133 (2013).

72. Lonskaya, I. et al. Diminished parkin solubility and co-localization with intraneuronal amyloid- $\beta$ are associated with autophagic defects in Alzheimer's disease. J. Alzheimers Dis. 33, 231-247 (2013).

73. Lukiw, W. J. Circular RNA (circRNA) in Alzheimer's disease (AD). Front. Genet. 4, 307 (2013).

74. Werfel, S. et al. Characterization of circular RNAs in human, mouse and rat hearts. J. Mol. Cell Cardiol. 98, 103-107 (2016).

75. Tan, W. L. W. et al. A landscape of circular RNA expression in the human heart. Cardiovasc. Res. 113, 298-309 (2017). 
76. Wang, $K$. et al. A circular RNA protects the heart from pathological hypertrophy and heart failure by targeting miR-223. Eur. Heart J. 37, 2602-2611 (2016).

77. Geng, H. H. et al. The circular RNA Cdr1as promotes myocardial infarction by mediating the regulation of miR-7a on its target genes expression. PLOS ONE 11, e0151753 (2016).

78. Li, B. et al. MicroRNA-7a/b protects against cardiac myocyte injury in ischemia/reperfusion by targeting poly(ADP-ribose) polymerase. PLOS ONE 9 e90096 (2014)

79. Wang, K. et al. Circular RNA mediates cardiomyocyte death via miRNAdependent upregulation of MTP18 expression. Cell Death Differ. 24 1111-1120 (2017).

80. Tondera, D. et al. The mitochondrial protein mtp18 contributes to mitochondrial fission in mammalian cells. J. Cell Sci. 118, 3049-3059 (2005).

81. Li, M. et al. A circular transcript of $n \subset x 1$ gene mediates ischemic myocardia injury by targeting miR-133a-3p. Theranostics 8, 5855-5869 (2018).

82. American Diabetes Association. Diagnosis and classification of diabetes mellitus. Diabetes Care 36, S67-S74 (2013).

83. Stoll, L. et al. Circular RNAs as novel regulators of $\beta$-cell functions in normal and disease conditions. Mol. Metab. 9, 69-83 (2018).

84. Xu, H., Guo, S., Li, W. \& Yu, P. The circular RNA Cdr1as, via miR-7 and its targets, regulates insulin transcription and secretion in islet cells. Sci. Rep. $\mathbf{5}$ 12453 (2015)

85. Shan, K. et al. Circular noncoding RNA HIPK3 mediates retinal vascular dysfunction in diabetes mellitus. Circulation 136, 1629-1642 (2017).

86. Panda A. C. \& Gorospe M. Detection and Analysis of Circular RNAs by RT-PCR. Bio Protoc. 8, e2775 (2018).

87. Salzman, J., Chen, R. E., Olsen, M. N., Wang, P. L. \& Brown, P. O. Cell-type specific features of circular RNA expression. PLoS Genet. 9, e1003777 (2013).

88. Song, Z. et al. Identification of urinary Hsa_circ_0137439 as a potential biomarker and tumor regulator of bladder cancer. Neoplasma 67, 137-146 (2020)

89. Wang, Y. et al. Exosomal circRNAs: biogenesis, effect and application in human diseases. Mol. Cancer 18, 116 (2019).

90. Fanale, D., Taverna, S., Russo, A. \& Bazan, V. Circular RNA in exosomes. Adv. Exp. Med. Biol. 1087, 109-117 (2018)

91. Li, Y. et al. Circular RNA is enriched and stable in exosomes: a promising biomarker for cancer diagnosis. Cell Res. 25, 981-984 (2015).

92. Li, S. et al. ExoRBase: a database of CircRNA, LncRNA and MRNA in human blood exosomes. Nucleic Acids Res. 46, D106-D112 (2018).

93. $\mathrm{Ma}, \mathrm{H}$. et al. Differential expression study of circular RNAs in exosomes from serum and urine in patients with idiopathic membranous nephropathy. Arch. Med. Sci. 15, 738-753 (2019).

94. Chen, X. et al. PRMT5 circular RNA promotes metastasis of urothelial carcinoma of the bladder through sponging MiR-30c to induce epithelial-mesenchymal transition. Clin. Cancer Res. 24, 6319-6330 (2018).

95. Vo, J. N. et al. The landscape of circular RNA in cancer. Cell 176, 869-881 (2019).

96. Chen, S., Li, T., Zhao, Q., Xiao, B. \& Guo, J. Using circular hsa_circ_0000190 as a new biomarker in the diagnosis of gastric cancer. Clin. Chim. Acta 466 167-171 (2017).

97. Li, P. et al. Using circular RNA as a novel type of biomarker in the screening of gastric cancer. Clin. Chim. Acta 444, 132-136 (2015).

98. Hang, D. et al. A novel plasma circular RNA CircFARSA is a potential biomarker for non-small cell lung cancer. Cancer Med. 7, 2783-2791 (2018).

99. Tan, S. et al. Circular RNA F-CircEA-2a derived from EML4-ALK fusion gene promotes cell migration and invasion in non-small cell lung cancer. Mol. Cancer 17, 138 (2018).

100. Zhu, K. et al. Plasma Hsa_circ_0027089 is a diagnostic biomarker for hepatitis B virus-related hepatocellular carcinoma. Carcinogenesis 41, 296-302 (2020).

101. Zuo, Z. et al. BBCancer: an expression atlas of blood-based biomarkers in the early diagnosis of cancers. Nucleic Acids Res. 48, D789-D796 (2020).

102. Li, Y. et al. Circular RNA expression profile of Alzheimer's disease and its clinical significance as biomarkers for the disease risk and progression. Int. J. Biochem. Cell Biol. 123, 105747 (2020).

103. Li, Y et al. Profiling of differentially expressed circular RNAs in peripheral blood mononuclear cells from Alzheimer's disease patients. Metab. Brain Dis. 35, 201-213 (2020)

104. Lu, D. \& Thum, T. RNA-based diagnostic and therapeutic strategies for cardiovascular disease. Nat. Rev. Cardiol. 16, 661-674 (2019).
105. Aufiero, S., Reckman, Y. J., Pinto, Y. M. \& Creemers, E. E. Circular RNAs open a new chapter in cardiovascular biology. Nat. Rev. Cardiol. 16, 503-514 (2019).

106. Vausort, M. et al. Myocardial infarction-associated circular RNA predicting left ventricular dysfunction. J. Am. Coll. Cardiol. 68, 1247-1248 (2016).

107. Zhang, J. et al. Plasma circular RNAs, Hsa circRNA 025016, predict postoperative atrial fibrillation after isolated off-pump coronary artery bypass grafting. J. Am. Heart Assoc. 7, e006642 (2018).

108. Holdt, L. M. et al. Circular non-coding RNA ANRIL modulates ribosomal RNA maturation and atherosclerosis in humans. Nat. Commun. 7, 12429 (2016).

109. Yang, P. et al. Silencing of CZNF292 circular RNA suppresses human glioma tube formation via the Wnt/ $\beta$-catenin signaling pathway. Oncotarget $\mathbf{7}$ 63449-63455 (2016).

110. Grumolato, L. et al. Canonical and noncanonical Whts use a common mechanism to activate completely unrelated coreceptors. Genes Dev. 24, 2517-2530 (2010).

111. Katoh, M. Canonical and non-canonical WNT signaling in cancer stem cells and their niches: cellular heterogeneity, omics reprogramming, targeted therapy and tumor plasticity (Review). Int. J. Oncol. 51, 1357-1369 (2017).

112. Bose, M., Almas, S. \& Prabhakar, S. Wnt signaling and podocyte dysfunction in diabetic nephropathy. J. Investig. Med. 65, 1093-1101 (2017).

113. Gay, A. \& Towler, D. A. Wht signaling in cardiovascular disease: opportunities and challenges. Curr. Opin. Lipidol. 28, 387-396 (2017)

114. Wang, Z., Liu, C. H., Huang, S. \& Chen, J. Wnt signaling in vascular eye diseases. Prog. Retin. Eye Res. 70, 110-133 (2019).

115. Harb, J., Lin, P. J. \& Hao, J. Recent development of wnt signaling pathway inhibitors for cancer therapeutics. Curr. Oncol. Rep. 21, 12 (2019).

116. Yang, J. et al. Guidelines and definitions for research on ephitelialmesenchymanl transition. Nat. Rev. Mol. Cell Biol. 21, 341-352 (2020).

117. Nieto, M. A., Huang, R. Y. J., Jackson, R. A. \& Thiery, J. P. EMT: 2016. Cell 166 21-45 (2016).

118. Sebio, A. \& Lenz, H. J. Molecular pathways: hippo signaling, a critical tumor suppressor. Clin. Cancer Res. 21, 5002-5007 (2015).

119. Peng, Q. S. et al. circRNA_0000140 suppresses oral squamous cell carcinoma growth and metastasis by targeting miR-31 to inhibit Hippo signaling pathway. Cell Death Dis. 11, 112 (2020).

120. Di Agostino, S. et al. YAP enhances the pro-proliferative transcriptional activity of mutant p53 proteins. EMBO Rep. 17, 188-201 (2016).

121. Feng, J. et al. Verteporfin, a suppressor of YAP-TEAD complex, presents promising antitumor properties on ovarian cancer. Onco Targets Ther. 9 5371-5381 (2016).

122. Sobhani, M., Abdi, J., Manujendra, S. N., Chen, C. \& Chang, H. PRIMA-1Met induces apoptosis in Waldenström's Macroglobulinemia cells independent of p53. Cancer Biol. Ther. 16, 799-806 (2015).

123. Jeck, W. R. et al. Circular RNAs are abundant, conserved, and associated with ALU repeats. RNA 19, 141-157 (2013).

124. Zhang, Y. et al. The biogenesis of nascent circular RNAs. Cell Rep. 15, 611-624 (2016).

125. Enuka, Y. et al. Circular RNAs are long-lived and display only minimal early alterations in response to a growth factor. Nucleic Acid Res. 44, 1370-1383 (2016)

126. Goodall, G. J. \& Wickramasinghe, V. O. RNA in cancer. Nat. Rev. Cancer 21 22-36 (2021).

127. Bachmayr-Heyda, A. et al. Correlation of circular RNA abundance with proliferation-exemplified with colorectal and ovarian cancer, idiopathic lung fibrosis, and normal human tissues. Sci. Rep. 5, 8057 (2015).

128. Szabo, L. \& Salzman, J. Detecting circular RNAs: bioinformatic and experimental challenges. Nat. Rev. Genet. 17, 679-692 (2016).

129. Li, H. M., Ma, X. L. \& Li, H. G. Intriguing circles: conflicts and controversies in circular RNA research. Wiley Interdiscip. Rev. RNA 10, e1538 (2019).

130. Bak, R. O. \& Mikkelsen, J. G. miRNA sponges: soaking up miRNAs for regulation of gene expression. Wiley Interdiscip. Rev. RNA 5, 317-333 (2014).

131. Piwecka, M. et al. Loss of a mammalian circular RNA locus causes miRNA deregulation and affects brain function. Science 357, eaam8526 (2017).

132. Hansen, T. B. et al. miRNA-dependent gene silencing involving Ago2mediated cleavage of a circular antisense RNA. EMBO J. 30, 4414-4422 (2011)

133. Costello, A., Lao, N. T., Barron, N. \& Clynes, M. Reinventing the wheel: synthetic circular RNAs for mammalian cell engineering. Trends Biotechnol. 38, 217-230 (2020). 
134. Ma, L. et al. Silencing of circRACGAP1 sensitizes gastric cancer cells to apatinib via modulating autophagy by targeting miR-3657 and ATG7. Cell Death Dis. 11, 169 (2020).

135. Xiong, D. D. et al. High throughput circRNA sequencing analysis reveals nove insights into the mechanism of nitidine chloride against hepatocellular carcinoma. Cell Death Dis. 10, 658 (2019).

136. Liu, Z. et al. CircRNA-5692 inhibits the progression of hepatocellular carcinoma by sponging miR-328-5p to enhance DAB2IP expression. Cell Death Dis. 10, 900 (2019).
137. Cao, L. et al. Circular RNA circRNF20 promotes breast cancer tumorigenesis and Warburg effect through miR-487a/HIF-1a/HK2. Cell Death Dis. 11, 145 (2020).

138. Rybak-Wolf, A. et al. Circular RNAs in the mammalian brain are highly abundant, conserved, and dynamically expressed. Mol. Cell 58, 870-875 (2015).

139. You, X. et al. Neural circular RNAs are derived from synaptic genes and regulated by development and plasticity. Nat. Neurosci. 18, 603-610 (2015). 